\title{
Detection of O-glycosylated proteins from different Trichinella species muscle larvae total extracts
}

\author{
R. MILCHEVA ${ }^{1,2^{*}}$, S. PETKOVA ${ }^{1}$, P. BABÁL ${ }^{2}$
}

${ }^{1}$ Institute of Experimental Pathology and Parasitology, Bulgarian Academy of Sciences, Acad. G. Bonchev Str., block 25, 1113 Sofia, Bulgaria, E-mail: rosicamilcheva@abv.bg; ${ }^{2}$ Department of Pathology, Faculty of Medicine, Comenius University, Sasinkova 4, 81108 Bratislava, Slovak Republic

\begin{abstract}
Summary
The aim of the work was to analyze oligosaccharide composition with the focus on O-linked glycoproteins presence in the total extract obtained from different Trichinella species muscle larvae by means of lectin affino-blot with lectins selected for their sugar specificity. The absence of reactivity with two lectins, Tritrichomonas mobilensis lectin and Maackia amurensis agglutinin, indicated that the species of the Trichinella genus do not synthesize sialic acid. Reactivity with Helix pomatia lectin, Vicia villosa lectin-B4, peanut agglutinin and Ulex europeus lectin-I identified the presence of O-linked glycans identical to carcinoma-associated Tn-antigen (GalNAc- $\alpha$-Ser/Thr) and T-antigen (Gal- $\beta 1,3-G a l N A c-\alpha-S e r / T h r)$ and also structures analogous to $\mathrm{ABH}-$ blood group antigens. The results obtained may contribute to a better understanding of the glycobiology of this parasitic nematode in relation to occupation of its intracellular niches.
\end{abstract}

Keywords: Trichinella, O-glycans, lectin-blot

\section{Introduction}

Glycoconjugates play a significant role in the life cycle and pathology of most major parasites. It is becoming clear that many of the protozoal and helminthic parasites rely on carbohydrate-binding proteins in the host to promote their parasitism, and they have elaborated intriguing strategies to defeat the anticarbohydrate immunity of the host (Schmidt \& Roberts, 1996).

The mechanisms by which infectious Trichinella larvae recognise, invade, and migrate within the intestinal epithelium are still unknown. However, it is likely that carbohydrate structures on the nematode contact surface and/or in its excretory/secretory products (ESP) are important in these events (Butcher et al., 2000). Trichinella glycosylated ESP had been found inside of the hypertrophic nuclei of nurse cells (Vassilatis et al., 1996; Yao \& Jasmer, 1998), raising the assumption that they could be involved in the induction of the aberrant skeletal muscle phenotype. It was shown however, that recombinant $49 \mathrm{kDa} \mathrm{ES}$ protein of $T$. nativa does not lose its antigenicity, although the recombinant form was deglycosylated. Moreover, it was reactive with five sera from mice infected with different Trichinella strains (Zheng et al., 2007). The glycobiology of Trichinella is becoming a subject of rising interest. N-glycosylation has been characterized in detail in adult $T$. spiralis total extracts (Morelle et al., 2000) and also in ESP from $T$. spiralis muscle larvae (Gruden-Movsesijan et al., 2002), in which the presence of the unusual sugar tyvelose has been discovered (Wisnewski et al., 1993; Reason et al., 1994). Except for one report (Gruden-Movesijan et al., 2002), there is virtually no information available on the structure and the synthesis of O-glycans in Trichinella. This work is focused on the demonstration of the mucin-type O-glycosylated proteins in muscle larvae crude extract from five different Trichinella isolates by a set of lectins selected according to their sugar specificity.

\section{Material and methods}

\section{Parasites and crude extract preparation}

The reference strains T. spiralis (code ISS03), T. pseudospiralis (code ISS13), T. britovi (code ISS02), T. murrelli (code ISS035) and T. nelsoni (code ISS029) used in this study were kindly provided by Prof. Eduardo Pozio (Instituto Superiore di Sanita, Italy). Briefly, BALB/C mice were inoculated with 400 infectious larvae of each isolate. Trichinella spp. muscle larvae were recovered 45 days post infestation (d.p.i.) by artificial digestion of muscle tissue of the carcasses according to a standard protocol, as referenced in Pozio et al. (1992) and Zarlenga et al. (1999). Muscle larvae of each isolate were washed three times with physiological saline solution, then homogenized in glassTeflon homogenizer in phosphate buffered saline, $\mathrm{pH} 7.2$ 
Table 1. Carbohydrate binding specificity of the lectins applied in this study

\begin{tabular}{cccc}
\hline Lectin & Abbreviation & $\begin{array}{c}\text { Carbohydrate binding } \\
\text { specificity }\end{array}$ & Concentration \\
\hline Helix pomatia agglutinin & HPA & GalNAc- $\alpha-$ & $20 \mu \mathrm{g} / \mathrm{ml}$ \\
Vicia villosa lectin-isoform B4 & VVL-B4 & GalNAc- $\alpha-$ & $20 \mu \mathrm{g} / \mathrm{ml}$ \\
Arachis hypogea agglutinin & PNA & Gal $\beta 3 \mathrm{GalNAc}$ & $50 \mu \mathrm{g} / \mathrm{ml}$ \\
Ulex europaeus agglutinin-I & UEA-I & Fuc $\alpha 2 \mathrm{Gal}$ & $50 \mu \mathrm{g} / \mathrm{ml}$ \\
Maackia amurensis lectin-II & MAL & Neu5Ac $\alpha 3 \mathrm{Gal} \beta 4 \mathrm{GlcNAc}$ & $6.6 \mu \mathrm{g} / \mathrm{ml}$ \\
Tritrichomonas mobilensis lectin & TML & Neu5Ac & $2.5 \mu \mathrm{g} / \mathrm{ml}$
\end{tabular}

(PBS), on an ice bath and centrifuged at $10000 \mathrm{~g} / \mathrm{min}$ for $30 \mathrm{~min}$. The supernatant was used as a crude extract and stored at $-80^{\circ} \mathrm{C}$. The protein content was determined by the method of Bradford (1976).

\section{SDS-PAGE and Western blot}

Components of the Trichinella crude extract products of all five isolates were separated on $10 \%$ SDS-polyacrylamide mini-gel electrophoresis under reducing conditions as described previously (Laemli, 1970) and then subjected to
Western blotting on nitrocellulose membranes (Sigma) according to Towbin et al. (1979). Membranes were blocked with $5 \%$ non-fat dry milk in tris-buffered saline (TBS), pH 7.2 for 30 min.

Blots were incubated with biotinylated lectins- Arachis hypogea agglutinin (PNA), Vicia villosa lectin, $\mathrm{B}_{4}$-isoform (VVL-B ${ }_{4}$ ) and Maackia amurensis lectin, type II (MAL-II, Vector Laboratories), Helix pomatia agglutinin (HPA) and Ulex europaeus agglutinin, type I (UEA-I, Sigma-Aldrich), and Tritrichomonas mobilensis lectin (TML, Calbiochem-
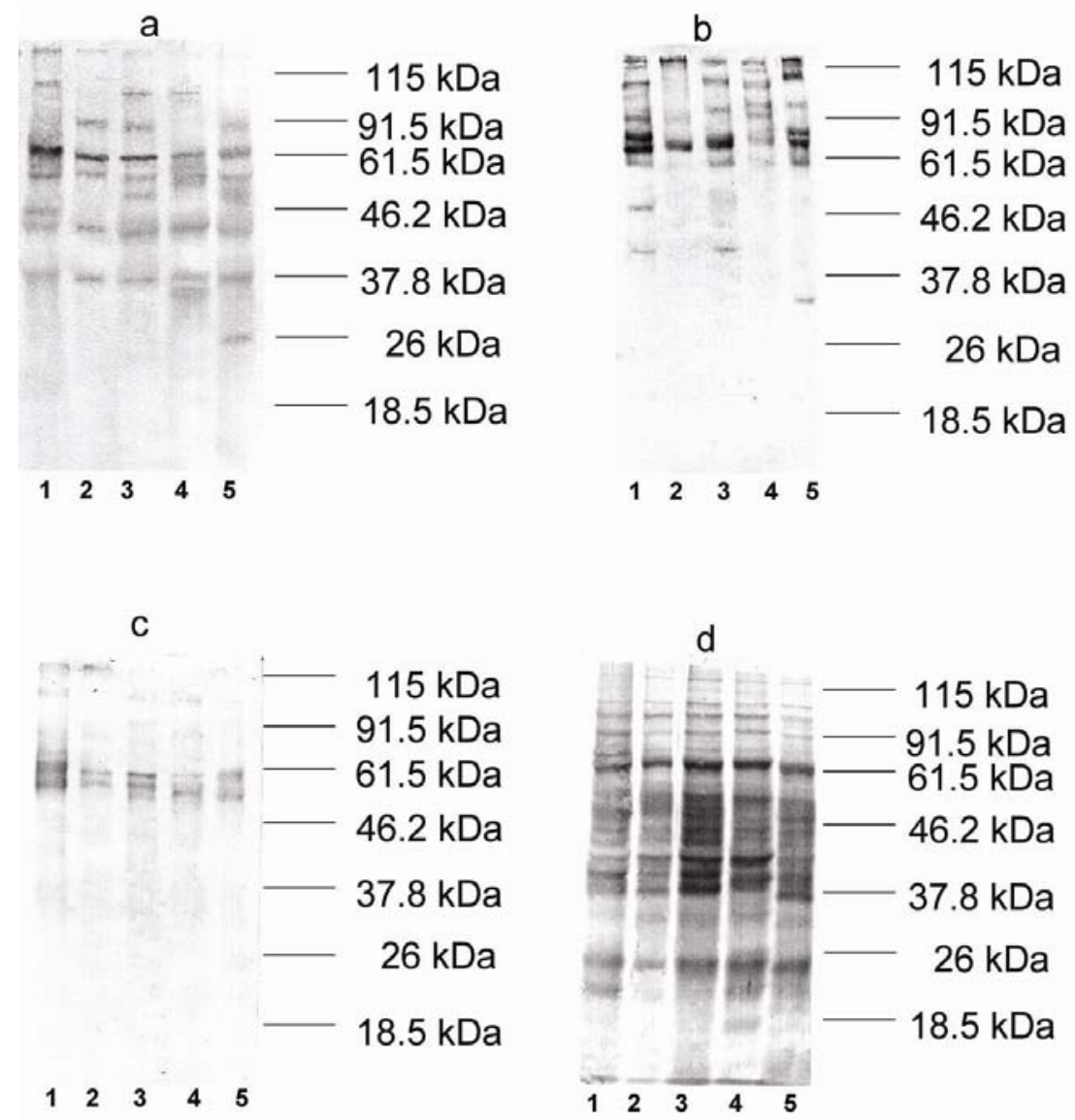

Fig. 1. Affino-blot analyses of Trichinella spp glycoproteins from muscle larvae total extracts, 45 d.p.i. The oligosaccharide sequence of the glycoproteins was probed with lectins of different carbohydrate specificity: a-VVL-B4; b-PNA; c-HPA; d-UEA-I. Specificity of binding was controlled by inhibition with appropriate sugars (data are not presented here). Line 1 T. nelsoni: 2 T. murrelli: 3 T. britovi: 4 T. pseudospiralis: 5 T. spiralis. 
Novabiochem). The sugar specificities and the concentrations used are listed in Table 1. The specificity of the binding was checked by inhibition of the particular lectin with $0.1 \mathrm{M}$ solution of the target monosaccharide: galactose for PNA, N-acetylgalactosamine for VVL-B $\mathrm{B}_{4}$ and HPA, fucose for UEA-I and sialic acid for TML (SigmaAldrich). The membranes were incubated with the lectins for 1 hour at room temperature, washed three times with TBS for $5 \mathrm{~min}$, then incubated $30 \mathrm{~min}$ with streptavidinHRP (Sigma-Aldrich) and washed three times with TBS. The color reaction was developed by $10 \mathrm{~min}$ incubation with $0.18 \%$ diamino benzidine solution (Dako Cytomation) (TBS pH 7.2, $0.05 \% \mathrm{H}_{2} \mathrm{O}_{2}$ ). The approximate molecular masses of the proteins identified by lectin-blot were estimated versus protein standards (Beijing Genetech Co., Ltd) ranging from 115 to $18.5 \mathrm{kDa}$.

\section{Results}

The proteins of the muscle larvae total extracts from different Trichinella isolates were separated in gels, which were stained with Coomassie brilliant blue. The pattern of the bands did not show remarkable differences between the particular strains. The pattern of crude extract glycoproteins, visualized by binding with different lectins, is shown in Fig. 1. All applied lectins, except for MAL-II and TML, showed sugar-specific reactions with Trichinella total extract products. The five Trichinella isolates share several bands of glycoproteins identified by HPA, VVL-B 4 , PNA and UEA-I, summarized in Table 2. Among the bands common for all strains, there was a glycoprotein with molecular mass of $58 \mathrm{kDa}$ that simultaneously reacted to HPA, VVL-B 4 and PNA, and another one of $111 \mathrm{kDa}$, which interacted with both PNA and UEA-I. The most abundant number of glycoproteins common to all strains was reactive with UEA-I. The greatest interspecies variety was displayed by the products identified with PNA.

\section{Discussion}

O-linked glycans are involved in cell-adhesion events during sperm-egg fertilization, host-microbial interactions and viral infections (Yang et al., 2000; Hooper \& Gordon, 2001; Primakoff \& Myles, 2002). A variety of tumor-associated antigens are O-linked glycans (Brockhausen, 1999). Recently, evidence was provided that Tn-antigen is widely distributed among the parasites belonging to both major phyla, the Plathelminthes and the Nemathelminthes (Casaravilla et al., 2003), and sialyl-Tn-antigen was particularly found in Echinococcus granulosus and Fasciola hepatica (Alvarez Errico et al., 2001; Freire et al., 2003).

Our study was focused on identifying the O-glycosylation in total extract proteins from members of the Trichinella genus. For this purpose we used the lectins VVL-B V $_{4}$ and HPA to demonstrate Tn-structures (Tollefsen \& Kornfeld, 1983; Roth, 1984), and PNA because of its specificity to T-antigen (Lotan, 1975). To determine whether these structures had been sialylated, we used also MAL-II and TML. The first lectin recognizes $\alpha-2,3$-linked sialic acid, and the second one identifies sialic acid residues without any linkage preference (Knibs et al., 1991; Babál et al., 1994). Finally, UEA-I was applied to indicate $\alpha 2$-bound Fuc (Sugii \& Kabat, 1982).

The results showed that glycoproteins bearing both Tnand T-antigens are abundantly present in somatic extracts of Trichinella spp (Fig. 1-a, b). T-antigen (Galß3GalNAc$\alpha$ Ser/Thr) derives after $\beta 3$-galactosylation of Tn-structure (GalNAc- $\alpha$ Ser/Thr) (Brockhausen, 1999). One glycoprotein with molecular mass of $58 \mathrm{kDa}$ was common to all isolates (Table 2) and simultaneously interacted with HPA, VVL-B ${ }_{4}$ and PNA. It seems that not all Tn- structures are good substrates for the $\beta 3$-galactosyltransferase in Trichinella because this $58 \mathrm{kDa}$ glycoprotein exposes T-antigens, illustrated by PNA reactivity, together with Tn-structures, which was shown through the interaction with HPA and

Table 2. Interaction of Trichinella spp. total extract products with lectins

\begin{tabular}{ccccccc}
\hline \multicolumn{6}{l}{ Molecular masses of proteins in the total extract of T. spiralis, T. pseudospiralis, T. britovi, T. murrelli and $T}$. \\
nelsoni $(\mathrm{kDa})$ which reacted in lectin-blot
\end{tabular}


VVL- $B_{4}$. This observation could be explained by the fact that the specificity of this enzyme is controlled by the amino acid sequence of the peptide substrates (Granovsky et al., 1994).

Obviously, sialyl-Tn and sialyl-T do not exist in Trichinella spp. since their somatic glycoproteins reacted neither with TML, nor with MAL-II. This was in accordance with the previous report on the absence of acidic sugars in adult T. spiralis (Morelle et al., 2000). Moreover, alcian-blue staining of $T$. spiralis muscle larvae tissue sections and histochemistry with the lectins specific for sialic acid also did not show any labeling (Milcheva et al., 2009). The absence of particularly $\alpha 6$-silaylation in $T$. spiralis muscle larvae ESP has been reported by Gruden-Movsesijan et al. (2002).

We used HPA in the context of its ability to recognize Tnantigen (Roth, 1984), a feature that resembles the VVL-B 4 affinity (Table 1). Both lectins showed the same pattern of staining on T. spiralis muscle larvae tissue sections (Milcheva et al., 2009). The lectin-blots however revealed that

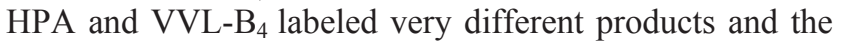
pattern of labeling was different as well (Fig. 1-a, c). Actually, only the $58 \mathrm{kDa}$ glycoprotein mentioned above interacted with both HPA and VVL-B 4 (Table 2), suggesting that Tn-glycans (GalNAc- $\alpha$ Ser/Thr) still are appropriate ligands for HPA. Another intriguing finding was the small number of glycoproteins labeled with HPA and they were common for all the isolates. Thus, the question cropped up to what structure HPA was reactve in Trichinella. This lectin is able to bind single $\alpha-\mathrm{N}$-acetylgalactosaminyl residues but it also can interact with $\alpha$ GalNAc residues located at the nonreducing terminus of O-glycosidically linked oligosaccharides (Roth, 1984). Blood group A antigens (GalNAc $\alpha 3(\alpha 2 \mathrm{Fuc}) \mathrm{Gal})$ (Brockhausen, 1999; Varki et al., 1999) possess appropriate ligands for HPA but we did not find any common bands labeled with HPA and UEA-I. Theoretically, core 5 (GalNAca3GalNAc) and core 7 (GalNAca6GalNAc) structures (Brockhausen, 1999; Varki et al., 1999) also of $111 \mathrm{kDa}$ (Table 2) and several glycoproteins specific for the particular isolates $(98,85,73,62$ and $52 \mathrm{kDa}$ ) (Table 3) that were labeled with both PNA and UEA-I. Herein, another question arose concerning the PNA combining site. This lectin is known to recognize T-antigen (Lotan et al., 1975). Terminal $\alpha 2$-fucosylation of T-antigen is a common modification resulting in type $3 \mathrm{H}$-blood group sequence (Fuca2Gala3GalNAc-Ser/Thr) (Brockhausen, 1999). Once this modification occurs, PNA is not able to interact with the penultimate Gal (Pereira et al., 1976). If we accept that PNA has an affinity for T-antigen only, this set of glycoproteins should expose both T-antigens and type $3 \mathrm{H}$-blood group antigens, suggesting that for some reason not all T-antigens are good substrates for $\alpha 2$-fucosyltransferase in Trichinella. On the other hand, Levroney et al. (2005) pointed in their report that PNA recognizes the Gal $\beta 3$ GalNAc sequence not only on T-antigen but also on core 2 O-glycans (Gal $\beta 3(\beta 6$ GlcNAc)GalNAc$\alpha$ Ser/Thr). In mammals, $\alpha 2$-fucosyltransferases compete with $\beta 3$ - and $\beta 6-\mathrm{N}$-acetylglucosaminyltransferases and the latter enzyme is responsible for the core 2 biosynthesis (Brockhausen, 1999; Varki et al., 1999). Further, $\beta 6-$ GlcNAc can serve as a substrate for type 1 or 2 chain building and then for type 1 or $2 \mathrm{ABH}$-blood group antigens respectively. In the light of these considerations it is tempting to assume that the set of glycoproteins listed in Table 3, express type 1 or 2 B-blood group determinants, which display ligands equally attractive to PNA and UEAI. Based on the results in this study, the synthesis of Ablood group antigen (GalNAc $\alpha 3(\alpha 2 \mathrm{Fuc}) \mathrm{Gal})$ is not much likely to occur in Trichinella since we did not find any common glycoproteins simultaneously stained with HPA and UEA-I. Concerning the fucosylation in Trichinella, both hypotheses proposed here should be further elucidated. Moreover, the fact that all Trichinella spp. share a large number of glycoproteins reactive with UEA-I (Table 2) suggests that these products could be implicated in a strategy for successful accommodation and survival in the host that is general for the genus.

Table 3. Molecular masses of glycoproteins in Trichinella spp. muscle larvae total extract (kDa) which were labeled with both PNA and UEA-I

\begin{tabular}{llccc}
\hline \multicolumn{1}{c}{98} & \multicolumn{1}{c}{85} & 73 & 62 & 52 \\
\hline T. pseudospiralis & T. spiralis & T. nelsoni & T. spiralis & T. spiralis \\
T. britovi & T. pseudospiralis & & T. nelsoni & \\
T. nelsoni & & & & \\
\hline
\end{tabular}

expose suitable ligands for HPA. However, we are currently not able to provide evidence that these oligosaccharides, which are less distributed in mammals, exist in Trichinella.

UEA-I was the lectin that interacted with the greatest number of glycoproteins in all five Trichinella isolates (Fig. 1d). We found a lot of specific products but the main set of them seemed to be common for the genus. This lectin is able to bind $\alpha 2$-linked fucose residues (Sugii \& Kabat, 1982), which are known to build the ABH-blood group antigens (Watkins, 1966; Varki et al., 1999). The blots showed one common glycoprotein with molecular weight
The aim of the presented work was to provide initial information about the O-glycosylated proteins in muscle larvae of five Trichinella isolates and the experiments were carried out 45 d.p.i., in the condition of asynchronous invasion. Briefly, the results showed that the mucin-type O-glycosylation is widely distributed in Trichinella spp. The presence of glycoproteins bearing Tn- and T-antigens and $\mathrm{ABH}-$ blood group sequences was demonstrated by their interaction with the lectins VVL- ${ }_{4}$, PNA and UEA-I, however Tn- and T-antigens were not sialylated. The biosynthesis of core 5 and core 7 structures is also expected due to HPA specific pattern of labeling. 


\section{Acknowledgments}

The authors wish to acknowledge Prof. E. Pozio for providing the isolates of T. spiralis (ISS03), T. pseudospiralis (ISS13), T. britovi (ISS02), T. murrelli (ISS035) and T. nelsoni (ISS029). This work was in part funded by the grants MU-L-1507/05 from the Bulgarian Ministry of Education and Science and APVT-20-025204 from the Slovak Ministry of Education.

\section{References}

Alvarez Errico. D., Medeiros A., Miguez M., CasaRavilla C., Malgor R., Carmona C., Nieto A., OsinaGA E. (2001): O-glycosylation in Echinococcus granulosus: identification and characterization of the carcinomaassociated Tn antigen. Exp. Parasitol., 98: 100 - 109

Babál P., Pindak F. F, Wells D. J., Gardner W. A. (1994): Purification and characterization of a sialic acidspecific lectin from Tritrichomonas mobilensis. Biochem. J., 299: $341-346$

BRADFORD M. M. (1976): A rapid and sensitive method for the quantification of microgram quantities of protein utilizing the principle of protein-dye binding. Anal. Biochem., 72: $248-254$

BRocKHAUSEN I. (1999) Pathways of O-glycan biosynthesis in cancer cells. Biochim. Biophys. Acta., 1473: $67-95$ Butcher B. A., Gagliardo L. F., ManWarren T., ApPleton J. A. (2000): Larvae-induced plasma membrane wounds and glycoprotein deposition are insufficient for Trichinella spiralis invasion of epithelial cells. Mol. Biochem. Parasitol., 107: 207 - 218

Casaravilla C., Freire T., Malgor R., Medeiros A., Osinaga E., Carmona C. (2003): Mucin-type O-glycosylation in helminth parasites from major taxonomic groups: evidence for widespread distribution of the Tnantigen (GalNAc-Ser/Thr) and identification of UDPGalNAc: polypeptide N-acetyltransferase activity. J. Parasitol., 89: 701 - 714

Freire T., Casaravilla C., Carmona C., Osinaga E. (2003): Mucin-type O-glycosylation in Fasciolla hepatica: characterization of carcinoma-associated Tn and sialyl-Tn antigens and evaluation of GDP-GalNAc: polypeptide Nacetylgalactosaminyltransferase activity. Int. J. Parasitol., 33: $47-56$

Granovsky M., Bielfeldt T., Peters S., Paulsen H., Meldal., Brockhausen J., Brockhausen I. (1994): UDPgalactose: glycoprotein-N-acetyl-D-galactosamine 3$\beta$-D-galactosyltransferase activity synthesizing O-glycan core 1 is controlled by the amino acid sequence and glycosylation of glycopeptide substrates. Eur. J. Biochem., 221: $1039-1046$

Gruden-MovsesiJan A., IliC N., SOFRINICMilosaVlJeVIC L. (2002): Lectin-blot analyses of Trichinella spiralis muscle larvae excretory-secretory components. Paras. Res., 88: $1004-1007$

Hooper L. V., Gordon J. I. (2001): Commensal hostbacterial relationship in the gut. Science., 292: 1115-1118
Knibbs R N, Goldstein I. J, Ratclife R. M, Shibuya N. (1991): Characterization of the carbohydrate binding specificity of the leukoagglutinin lectin from Maackia amurensis. Comparison with the other sialic acid-specific lectins. J. Biol. Chem., 266: $83-88$

LAEMLY U. K. (1970): Cleavage of structural proteins during the assembly of the head of bacteriophage T4. $\mathrm{Na}$ ture, 227: $680-685$

Levroney E. L., Aguilar H. C., Fulcher J. A., Kohatsu L., Pace K. E., Pang M., Gurney K. B., Baum L. G., LEE B. (2005): Novel innate functions for galectin1: galectin-1 inhibits cell fusion by Nipah Virus envelope glycoprotein and augments dendritic cell secretion of proinflammatory cytokines. J. Immunol., 175: 413 - 420

Lotan R., SkUtelsky, E., DANON, D., SARON, N. (1975): The purification, composition and specificity of the anti-T lectin from peanut (Arachis hypogaea). J. Biol. Chem., 250 (85): $18-23$

Milcheva R., Petkova S., Dubinský P., HuRníková Z., BABÁL P. (2009): Glycosylation changes in different developmental stages of Trichinella. Biologia, 64 (1): 180-186 Morelle W., Haslam S. M., Morris H. R., Dell A. (2000): Characterization of the N-linked glycans of adult Trichinella spiralis. Mol. Biochem. Parasitol., 109: 171 177

Pereira M. E., Kabat E. A., Lotan R., Sharon N. (1976): Immunochemical studies on the specificity of the peanut (Arachis hypogaea) agglutinin. Carboh. Res., 51: $107-118$

Pozio E., La Rosa G., Rossi P., Murrell K. D. (1992): Biological characterization of Trichinella isolates from various host species and geographical regions. $J$. Parasitol., 78: 647 - 653

Primakoff P., Myles D. G. (2002): Penetration, adhesion and fusion in mammalian sperm-egg interaction. Science, 296: $2183-2185$

Reason A. J., Ellis L. A., Appleton J. A., Wisnewski N., Grieve R. B., McNeil M., WAsSom D. L., Morris H. R., Dell A. (1994): Novel tyvelose-containing tri- and tetra-antennary $\mathrm{N}$-glycans in the immunodominant antigens of the intracellular parasite Trichinella spiralis. Glycobiology, 4: $593-603$

Roth J. (1984): Cytochemical localization of terminal Nacetyl-G-galactosamine residues in cellular compartments of intestinal goblet cells: implications for the topology of O-glycosylation. J. Cell. Biol., 98: 399 - 406

SCHMIDT, G. D., RoBERTS L. S. (1996): Foundations of parasitology, $5^{\text {th }}$ edition. Wm. C. Brown, New York

SugII S., KABAT E. A. (1982): Further immunochemical studies on the combining sites of Lotus tetragolbulus and Ulex europaeus I and II lectins. Carbohydr. Res., 99: 99 101

Tollefsen S. E, KoRnfeld R. (1983) The B4 lectin from Vicia villosa seeds interacts with $\mathrm{N}$-Acetylgalactosamine residues $\alpha$-linked to serine or threonine residues in cell surface glycoproteins. J. Biol. Chem., 258: 5172 - 5176

Towbin H., Stabelin T., Gordon J. (1979): Electrophoretic transfer of protein from polyacrylamide gels to nitro- 
cellulose sheets. Proc. Natl. Acad. Sci. USA, 76: $4350-$ 4354

Varki A., R. D. Cummings, J. Esko, H. Freeze, G. HART, J. D. MARTH O-Glycans-Less common O-glycan core subtypes. Structures common to different types of glycans-The A, B, and $\mathrm{H}$ blood group structures. In: Essentials of Glycobiology. A. R. D. VARKI, J. CUMMINGS, H. Esko, G. Freeze, J. D. Hart, J. Marth (Eds), Cold Spring Harbor Laboratory Press, Cold Spring Harbor, NY, 1999. p. $232-239$

Vassilatis D. K., Polvere R. I., Despommier D. D., Gold A. M., VAn der Ploeg L. H. (1996): Developmental expression of a 43-kDa secreted glycoprotein from Trichinella spiralis. Mol. Biochem. Parasitol., 78:13 - 23

WATKINS W. M. (1966): Blood-group substances. Science., 152: $172-181$

WisnewsKi N., MCNeIL M., GRIEVE R. B., Wassom D. L. (1993): Characterisation of novel fucosyl- and tyvelosylcontaining glycoconjugates from Trichinella spiralis mus- cle stage larvae. Mol. Biochem. Parasitol., 61: 25 - 36

Yang Z. Y., Duckers H. J., Sullivan N. J., SANCheZ A., NABEL E. G., NABEL G. J. (2000): Identification of the Ebola virus glycoprotein as the main viral determinant of vascular cell cytotoxicity and injury. J. Nat. Med., 6: $886-$ 889

YAO C., JASMER D. P. (1998): Nuclear antigens in Trichinella spiralis infected muscle cells: nuclear extraction, compartmentalization, and complex formation. Mol. Biochem. Parasitol., 92: $207-218$

Zarlenga D. S., M., Chute B., Martin A., Kapel C. M. O. (1999): A multiplex PCR for unequivocal differentation of all encapsulated and non-encapsulated genotypes of Trichinella. Int. J. Parasitol., 29: 1859 - 1867

Zheng B. L., L. H. XIAO, X. R. WANG, D. M. LI, Y. X. LU, Y. Zhang, Q. B. Yan, M. X. Song. (2007): Study of the $49 \mathrm{kDa}$ excretory-secretory protein gene of Trichinella nativa and Trichinella spiralis. Helminthologia, 43: 120 125

ACCEPTED FEBRUARY 27, 2009 